Revista Interdisciplinaria de Estudios de Género de El Colegjo-de México-6, -dossier Género y Trabajo, e405

http://dx.doi.org/10.24201/reg.v6i0.405

Artículo

Dossier Género y Trabajo

\title{
Mujeres trabajadoras en Japón: perspectivas institucionales. demográficas y jurídicas de la desigualdad laboral
}

Women workers in Japan: institutional, demographic and legal perspectives of labor inequality

Fernando Villaseñor Rodríguez

Centro de Estudios de Asia y África de El Colegio de México

e Investigador en la Escuela Libre de Derecho. Ciudad de México, México.

Correo electrónico: fvillasenor@colmex.mx. (1) https://orcid.org/0000-0001-9676-730X

\section{Resumen}

Recibido: abril de 2019

Aceptado: noviembre de

2019

Publicado: enero de 2020

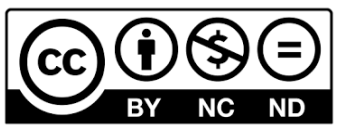

Esta obra está protegida bajo una Licencia Creative Commons Atribución-NoComercial-

SinDerivadas 4.0 Internacional (CC BY-NC-ND 4.0)
Este artículo se propone revisar la situación laboral de la mujer en Japón a partir de cuatro componentes: los antecedentes - a partir de la construcción de la familia tradicional y el papel de la mujer en ésta—; el diseño institucional — conforme con el estado de bienestar residual denominado "familismo"-; la coyuntura demográfica —que implica una baja tasa de natalidad y segregación laboral— y; el marco jurídico — compuesto por normas ineficaces e incoercibles-. Después de la revisión propuesta, se argumentará que los cambios en la legislación y las políticas públicas para alcanzar la igualdad laboral en Japón son y seguirán siendo insuficientes si no se toman en cuenta los cuatro componentes en su conjunto. Finalmente, se esbozarán algunas perspectivas de solución y posibles escenarios para el futuro próximo.

CÓMO CITAR: Villaseñor, Fernando. (2020). Mujeres trabajadoras en Japón: perspectivas institucionales, demográficas y jurídicas de la desigualdad laboral. Revista Interdisciplinaria de Estudios de Género de El Colegio de México, 6, e405, dossier Género y Trabajo. doi: http://dx.doi.org/10.24201/reg.v6i0.405

http://estudiosdegenero.colmex.mx 
Palabras clave: Japón; trabajo; equidad de género; estado de bienestar social; derecho.

\begin{abstract}
This article aims to review the employment conditions of women in Japan according to four components: the historical background -from the construction of the traditional family and the role of women in it-, the institutional design — in accordance with the residual welfare state called "familism"-, the demographic conjuncture — implying a low birth rate-, and the legal framework-formed by ineffective and incoercible rules. After the review, it will be argued that changes in legislation and public policies to achieve labor equality in Japan are, and will remain, insufficient if they do not consider the four components as a whole. Finally, some solution prospects and possible scenarios for the near future will be outlined.
\end{abstract}

Keywords: Japan; work; gender equality; welfare state; law.

\title{
Introducción
}

A mediados del 2018 el Ministerio de Salud, Trabajo y Bienestar de Japón anunció que, conforme a su último censo, la tasa de participación femenina en el empleo había llegado al 70\% (Ministry of Health Labor and Welfare, 2018). En medios de información y discursos oficiales, el gobierno encabezado por el Primer Ministro Shinzo Abe proclamó el triunfo de los "womenomics" y de la "iniciativa para lograr una sociedad en la que todas las mujeres brillen" (traducción propia) (Gender Equality Bureau, 2017) .

\footnotetext{
${ }^{1}$ Womenomics es una serie de políticas públicas y económicas basadas en la idea de que el avance económico de las mujeres mejorará la economía japonesa en su conjunto. Desde el anuncio de Shinzo Abe en 2015 se han fijado entre otros objetivos, para 2020, incorporar al 73\% de las mujeres de 25 a 44 años en la fuerza laboral, aumentar el número de mujeres en puestos ejecutivos en empresas privadas y crear nuevos espacios para
} 
No obstante, pocos meses más tarde, los rumores sobre la alteración en los exámenes de ingreso para limitar el número de mujeres en la Facultad de Medicina de la Universidad de Tokio - entre otras muchas universidades - se volvieron una vergonzosa realidad (Yajima, 2018). Un escándalo similar casi le cuesta el puesto a Abe cuando en 2019 se supo que se habían manipulado cifras sobre empleos, salarios y pensiones, siendo las mujeres las principales afectadas (The Asahi Shimbun, 2019). ¿Cómo se puede explicar esta contradicción entre empoderamiento y desigualdad en un país que se considera la tercera economía del mundo y uno de los estándares del desarrollo?

Se argumentará que, a partir de construcciones ideológicas y arquetipos disfrazados de "tradición” y "cultura", se han justificado modelos sociales, jurídicos y laborales que privilegian una versión monolítica de la familia y, con ella, del papel de la mujer. Esto coincide con los postulados generales que Eric Hobsbawm llama "la invención de la tradición" (Hobsbawm, 2012) y, más específicamente, Chizuko Ueno denomina la "trampa de la familia tradicional" (Ueno, 2008, p. 63). Al considerar un modelo específico de familia como "tradicional", y al explicar la discriminación como "una práctica de la cultura", se legitima el estado de cosas como algo natural e inmutable. Bajo este razonamiento, la desigualdad no sería una patología, sino un atributo de lo "propiamente japonés".

Evidentemente, este razonamiento no es correcto o deseable. Por ello, aunque este texto reconoce el trasfondo no-occidental, asiático, confuciano y específicamente japonés, busca evitar las explicaciones fundadas en el determinismo cultural. El fenómeno de la discriminación laboral hacia las mujeres es complejo y, en efecto, incluye particularidades que dependen de su contexto. Sin embargo, si no pudieran identificarse también generalidades y patrones nocivos dentro de una cultura, cualquier análisis resultaría una mera repetición de dogmas. Debido a ello, es posible tener una posición crítica que al mismo tiempo sea respetuosa de una cultura; este texto se presenta desde tal posición.

guarderías, así como políticas laborales adecuadas para madres trabajadoras. Al momento de escribir esto los resultados han sido poco alentadores (Gender Equality Bureau, 2017). 
Este artículo se propone analizar los antecedentes, diseño institucional, circunstancias demográficas y marco jurídico que han generado - y mantenido — la discriminación laboral de las mujeres en Japón. A partir de esta visión integral no solamente se podrá demostrar la falta de congruencia entre las versiones oficiales con la realidad, sino que también se podrá explicar por qué muchas veces estas inconsistencias pasan inadvertidas por la sociedad japonesa. En este sentido, se concluirá que las políticas, normas e instituciones públicas no han podido, ni podrán, reducir la brecha de género si siguen pasando por alto los diferentes componentes de este problema, así como sus combinaciones.

\section{Antecedentes}

A pesar de que muchos discursos e historias oficiales tratan de establecer un antiquísimo e inmutable papel de la mujer japonesa como ama de casa, esta noción es relativamente moderna. Durante siglos, las mujeres en Japón se han desempeñado, por voluntad o necesidad, en todo tipo de trabajos remunerados fuera del hogar. Es desde el periodo Meiji (1868-1912), con la industrialización acelerada y la imitación de las prácticas europeas y norteamericanas, que se empieza a construir la figura de la familia "tradicional" (Murakami, 1984). En Japón esta construcción recibe la denominación de “familia/hogar” 家 (ie), e incluye el correlativo mito de la mujer japonesa como inveterada "buena madre/esposa sabia" 良妻賢母 (Ryōsaikenbo) (Fujimura-Fanselow, 1991).

Aunque las razones por las cuales se inventa la ie son múltiples, tienen en común la intención de crear una visión homogénea sobre la conformación familiar y social en Japón. En este sentido, así como el emperador era considerado descendiente de los dioses y a su vez "padre de todos los japoneses”, así también en cada hogar había un “jefe de familia” 戸主 (koshu) que a partir del periodo Meiji prácticamente se equiparó al hombre con mayor edad (Hozumi, 1912, p. 85).

El koshu debía encargarse de proveer el sustento y decidir sobre los aspectos más relevantes en las vidas de todos los demás miembros de la ie. En esta lógica, la mujer debía apoyar al 
koshu, primero como madre, en la crianza, alimentación y cuidado de los hijos, y luego como esposa, ejecutando las instrucciones del koshu para conservar el prestigio de la $i e$.

La construcción de la ie se solidificó jurídicamente con el primer Código Civil Japonés de 1898, también conocido como Código Civil Meiji. Éste fue inicialmente inspirado por el Code Napoléon francés, pero después de casi diez años de protestas académicas que lo acusaban de ser demasiado liberal, se rehízo completamente conforme al Bürgerliches Gesetzbuch —o BGB - (Steenstrup,1991). El Código Civil Meiji se adhirió entonces casi en su totalidad a este nuevo modelo prusiano salvo en el apartado de familia.

Ya desde su título, "de la ie" 家に (ie ni), el Libro IV del Código Civil Meiji anunciaba que la regulación de las relaciones familiares se haría conforme con la "familia tradicional japonesa". En términos de derechos y obligaciones, esto implicaba un koshu casi omnipotente que controlaba la vida y los bienes de todos los demás miembros de la ie (Röhl, 2005). También limitaba la designación de herederos dándole preferencia al primogénito varón o varones subsecuentes y permitiendo en su defecto "adoptar" un esposo en caso de que solamente se tuvieran hijas (Mizubayashi, 1987). Estas normas se mantuvieron válidas hasta la derrota de Japón en la Segunda Guerra Mundial, e implicaron la legitimación de un sistema inequitativo y que le dio el control de las relaciones familiares a los hombres de mayor edad.

No obstante, tras la derrota japonesa el general Douglas MacArthur, quien lideraba el Ejército de Ocupación, se encargó de promover reformas sustantivas que desmantelaran las instituciones jurídicas anteriores a la guerra (Maki, 1968, p. 9). En primer lugar, se creó una nueva constitución que, a diferencia de la antigua Constitución del Imperio de Japón de 1868, reconocía la soberanía popular y dejaba al emperador como una figura meramente protocolaria.

Asimismo, en la nueva Constitución de Japón se añadió, en el artículo 14 constitucional, un reconocimiento del principio de igualdad en los siguientes términos: "Todos los ciudadanos son iguales ante la ley y no existirá discriminación política, económica o social por razones de raza, credo, sexo, condición social o linaje...” (Constitución,1947, art. 24). 
Pero dada la desigualdad institucionalizada por medio de la ie, se requería una regulación más específica que rompiera con el patriarcado del koshu y reconociera un sitio digno para las mujeres japonesas. Esta tarea se le encargó a Beate Sirota Gordon, una mujer que había fungido como intérprete para el Ejército de Ocupación y conocía la realidad de las mujeres japonesas al haber vivido en ese país durante su infancia y parte de su adolescencia (Sirota, Pharr, Hastings y Molony, 1988). El resultado de su trabajo se cristalizó en el artículo 24 de la Constitución de Japón que indica:

El matrimonio estará basado solamente en el mutuo consentimiento de ambos contrayentes y será mantenido por la cooperación mutua con igualdad de derechos de marido y mujer.

Las leyes que se dicten relativas a la elección del cónyuge, derechos de propiedad, herencia, elección de domicilio, divorcio y otros asuntos referentes al matrimonio o a la familia, tendrán en cuenta primordialmente la dignidad individual y la igualdad esencial de derechos de ambos sexos (Constitución, 1947, art. 24).

Este artículo no tenía precedente en Japón y se promulgó con el objetivo de desarticular jurídicamente al sistema de la ie.

Como consecuencia directa de los artículos 14 y 24 de la Constitución de Japón, hubo una reforma amplia y sustancial al Código Civil Japonés. Quizás el punto más trascendente de la reforma de posguerra, fue que el nuevo código eliminó por completo los Libros IV y V que regulaban a la ie en su conjunto (Nakamura, 1962). En su lugar, el nuevo Código Civil de 1948 establecía un Libro “sobre la familia” 家族について (kazoku ni tsuite) diametralmente distinto al del Código Civil Meiji. En concordancia con la Constitución de 1947, el nuevo Libro 4 se basó en la libertad de las partes para contraer matrimonio, así como la igualdad entre sexos dentro de las relaciones familiares. 
Empero, como señala Ueno "es importante distinguir los cambios legales de la práctica social en este tema" (Ueno, 2008, p. 65). Aunque ya no existía una obligación legal de patrilinealidad y primogenitura, las sucesiones han seguido aplicando sus principios varias décadas después de las reformas. Lo mismo ocurre tratándose de la propiedad, donde la mujer requirió hasta muy recientemente autorización de su esposo o padre para enajenar bienes y abrir una cuenta bancaria. Es decir, la costumbre ha dejado una impronta superior al derecho positivo y esto puede identificarse también en el tema del trabajo.

\section{Estado residual y trabajo}

Desde su formación moderna, el Estado japonés ha diseñado y gestionado sus políticas de bienestar social conforme con el modelo residual. Richard Titmuss define el modelo residual de bienestar como "aquel en el que los servicios sociales forman una red de seguridad bajo el sistema económico, y solo cuando los canales naturales de bienestar — el mercado privado y la familia - se derrumban, podría tener lugar el bienestar social, e incluso entonces, solo temporalmente" (Titmuss, 2008, pp. 30-31).

Es importante reconocer que la distribución del trabajo no remunerado es un componente de la división sexual del trabajo y no es exclusivo del caso japonés. En todas partes, en mayor o menor grado, el trabajo doméstico y de crianza es más comúnmente realizado por las mujeres, mientras que los hombres realizan un trabajo más propiamente remunerado de acuerdo con el modelo de sostén familiar basado en el hombre ${ }^{2}$ (OECD, 2012). Sin embargo, en Japón se han dado características específicas que han limitado diversos arreglos en esta distribución del trabajo.

Así, por ejemplo, Japón presenta una sub-especie del modelo residual de bienestar denominado "familismo" con dos componentes principales. En primer lugar, las empresas se

\footnotetext{
${ }^{2}$ Tampoco es del todo correcto hablar del modelo de sostén familiar basado en el hombre (male breadwinner model) ya que han existido múltiples variaciones sobre este modelo en diferentes historias nacionales (Lewis, 1992).
} 
adaptan a la dimensión familiar con "empleos de por vida" y aumentos salariales por antigüedad, mismos que reflejan los cambios en las obligaciones familiares a lo largo del ciclo de vida. En segundo lugar, existe una intervención pública limitada en asuntos familiares, incluyendo lo referente a la participación laboral y al trabajo no remunerado (Tachibanaki, 2010). Como se describirá a continuación, ambos componentes comparten un sesgo de género que favorece desproporcionadamente a los hombres.

Por lo que hace a la adaptación de las empresas conforme a la lógica familista, Kazuo Yamaguchi y Yoshio Higuchi argumentan que "las empresas japonesas basan los salarios no solo en los trabajadores varones sino también en sus esposas e hijos, como si contrataran a toda la familia del trabajador" (Yamaguchi y Higuchi, 2008, p. 23). Además, los hombres asalariados cuyas esposas no trabajan fuera del hogar por lo general se oponen a cambiar este sistema y tienen un enorme poder en sus empresas, en el gobierno y en los sindicatos (Yamaguchi y Higuchi, 2008). Esta mezcla entre prácticas empresariales, laborales y sociales sientan las bases para un modelo de empleabilidad preferencial hacia los hombres.

Por lo que hace al "empleo de por vida", conforme al cual se intercambia la movilidad laboral a cambio de estabilidad en el empleo, en teoría fue la base que conformó la economía japonesa para levantarse de las cenizas después de la Segunda Guerra Mundial. En realidad, sin embargo, este sistema es "dependiente de un rápido y constante crecimiento económico y una fuerza laboral joven". (Mari, 2012, p. 71). Tales circunstancias solamente existieron en el periodo inmediato a la posguerra, cuando hubo falta de mano de obra calificada, exceso de mano de obra no calificada, rápida transformación tecnológica y ausencia de sindicatos fuertes que pudieran limitar a una economía dirigista (Johnson, 1982).

Pero aun suponiendo la viabilidad contemporánea del sistema de "trabajo de por vida", el hecho de que requiera una alta inversión en tiempo para desarrollar habilidades específicas para cada compañía hace muy difícil, cuando no imposible, cambiarse de trabajo o reingresar al mercado laboral. Este factor es el que lleva a que la movilidad laboral en Japón sea de las más bajas internacionalmente (Ranstad Workmonitor, 2018). Asimismo, este factor es determinante para que las mujeres, cuando suspenden su trayectoria laboral para cuidar de 
niños o ancianos, no puedan regresar a sus lugares de trabajo o tengan que hacerlo perdiendo su antigüedad y jerarquía.

Otro factor de desigualdad laboral es el uso empresarial del tiempo extra no remunerado para reducir costos y evitar, o al menos reducir, los despidos. En Japón, los empleadores proveen estabilidad en el empleo para trabajadores de cuello blanco, pero a cambio les exigen horas "flexibles" de trabajo, un eufemismo que oculta horas extras ilimitadas y trabajo en días de descanso por periodos prolongados. Este arreglo es el que se ha denominado "flexiguridad" (Wilthagen y Tros, 2004), un balance entre seguridad en el empleo y flexibilidad de las condiciones laborales.

La flexiguridad en Japón funcionó como una válvula que evitó los despidos masivos durante varias décadas. Sin embargo, tras el estallido de la burbuja económica en 1990 y durante las tres décadas de recesión subsecuentes, resultó insuficiente. Aunque por un lado se intentó seguir protegiendo a los empleados de cuello blanco y a aquellos que ya tenían antigüedad, se liberalizó la regulación de los trabajos no regulares y con ello las nuevas contrataciones de trabajadores (Kamimura y Soma, 2013).

Con el fin de enfrentar la crisis económica, las empresas japonesas aumentaron significativamente la contratación de nuevos trabajadores bajo la categoría de no regulares. Esta práctica se generalizó a tal punto que extralegalmente se establecieron dos vías paralelas de trabajo, ascenso y capacitación en el sector privado: los trabajadores de la 総合職 sogoshoku — vía del trabajador integral—, lo que implica trabajo de por vida y flexiguridad; y los trabajadores de la —般職 ippanshoku — vía del asistente general一, lo que significa trabajo limitado en ascensos, condiciones laborales y horas de trabajo (Bishop, 2012). Mientras que la sogoshoku incluye principalmente trabajadores regulares, la ippanshoku incluye principalmente trabajadores no regulares.

En este sentido, es relevante destacar que un gran número de ippanshoku son mujeres y han servido para proteger la estabilidad en el empleo de sus colegas hombres. Esto, debido a que se argumenta que son los hombres quienes tienen la responsabilidad principal de llevar el 
sustento al hogar (Kasza, 2011). En términos porcentuales, la Agencia Nacional de Impuestos encontró que de 1992 a 2015 la tasa de empleo regular para mujeres bajó de 62 a 49.6\% (Ministerio del Interior, 2015). Comparativamente, la tasa de empleo regular para hombres bajó de 81 a 70.3\% en el mismo periodo (Ministerio del Interior, 2015).

Mientras tanto, en el mismo censo de 2015, los ingresos anuales de las trabajadoras no regulares promediaron 1,47 millones de yenes, muy por debajo de la línea de pobreza de 2 millones de yenes. Por otro lado, los hombres en empleos no regulares ganaron 2,2 millones de yenes al año, por encima de la línea de pobreza (Ministerio del Interior, 2015). Es decir, la disparidad entre trabajo regular y no regular ha sido un elemento que ha exacerbado la desigualdad de género.

\section{Empleo y diseño institucional}

La situación antes descrita no es producto ni de la casualidad ni — como el gobierno y algunos académicos japoneses argumentan — de la cultura japonesa, sino que implica un diseño institucional por parte del Ministerio de Salud, Trabajo y Bienestar y de la Agencia Nacional de Impuestos. A través de sus políticas fiscales y de pensiones, el Estado japonés promovió que las mujeres se dedicaran principalmente a labores domésticas y de cuidado y que, en caso de trabajar fuera del hogar, lo hicieran solamente a tiempo parcial.

En materia fiscal, desde 1961 se establecieron las "deducciones familiares" con el supuesto propósito de ayudar a las familias donde ambos esposos trabajan. La deducción implicaba la reducción proporcional del impuesto al salario por unidad familiar en vez de individualmente. En este sentido, el monto de la deducción disminuye en proporción a que el salario de uno de los esposos aumenta (Yokoyama, 2018). ${ }^{3}$

\footnotetext{
${ }^{3}$ Así, por ejemplo, si uno de los esposos gana menos de $¥ 700,000$ anuales hay una excepción para el total de ingresos por salario por familia de hasta $38 \%$ (más de la mitad queda exenta). Sin embargo, a partir de que va aumentando el ingreso de uno de los esposos, la cantidad exenta de impuestos disminuye; por ejemplo, si uno de los esposos gana anualmente $¥ 800,000$ sólo queda exento el $18 \%$ y cuando el ingreso anual es de $¥ 1,000,000$ yenes o más la cantidad de impuestos exenta (por familia) se reduce a cero. Esta es una de las razones por las que se desincentivan salarios superiores al millón de yenes para las mujeres, siempre es deseable que uno de
} 
Para efectos prácticos, como el salario del hombre en Japón, y en general en el mundo, ha sido generalmente superior al de la mujer, es común y en cierta forma lógico buscar la mayor deducción fiscal reduciendo, o incluso eliminando, el salario devengado por ésta última. El efecto es que un importante grupo de mujeres opten por no reinsertarse en el mercado laboral tras el matrimonio. Aunque las deducciones familiares se reformaron - primero en 2004 y más recientemente en 2018 - , con el fin de volver a incentivar la participación laboral femenina, diversos estudios comprueban que ha tenido resultados muy limitados (Akabayashi, 2006; Takahashi, 2010; Bessho y Hayashi, 2014).

En el mismo sentido, el “sistema nacional de pensiones” 国民年金 (kokumin nenkin), a partir de su reforma de 1985, exime a todas las mujeres casadas de pagar sus contribuciones para el fondo nacional de pensión. Las mujeres casadas, al llegar a la edad de jubilación, y aun cuando no hayan cotizado las semanas de trabajo necesarias $-\mathrm{o}$, aunque no hubieren trabajado fuera del hogar en absoluto - reciben la totalidad del monto pensionable a expensas del resto de los contribuyentes. Esta excepción en el pago de contribuciones, sin embargo, no aplica a mujeres solteras y, hasta muy recientemente, tampoco aplicaba a divorciadas (Osawa, 2013).

Dicho de otra forma, por lo que hace a impuestos y pensiones, el sistema japonés está configurado para ofrecer una ventaja significativa a las mujeres casadas que no trabajan fuera del hogar. Pero hay que hacer una distinción adicional, ya que este estatus de privilegio no favorece a todas las esposas japonesas, sino solamente a aquellas que forman parte de familias con ingresos medios y altos.

En efecto, un estudio demostró que en el 70\% de las familias donde la esposa solicitó la excepción de contribuciones para el fondo de pensión, el ingreso del esposo excedía los 9 millones de yenes anuales —alrededor de 1.5 millones de pesos a mitad del 2019. Correlativamente, estas mujeres en su mayoría renunciaron a sus trabajos al tener a su primer hijo y, al momento de considerar reincorporarse al mercado laboral, solo tuvieron la opción

los esposos perciba ingresos por salario menores a esa cantidad con el fin de poder optar por las deducciones familiares respecto del total. 
de trabajar como empleadas de medio tiempo (Nemoto, 2016). Haciendo un análisis costobeneficio, y debido a las exenciones fiscales y de pensiones, resultaba prácticamente igual, o en algunos casos incluso contraproducente, reingresar al mercado laboral. Conforme con ello, estas esposas optaron en su mayoría por vivir de un solo ingreso dependiente completamente del esposo.

Aun así, con todo lo criticable que pueda ser este diseño institucional por reconocer un solo modelo de familia y favorecer la dependencia económica de la mujer, no resulta aplicable para las familias de ingresos bajos. En las familias donde el esposo tiene ingresos menores a la línea de pobreza de 2 millones de yenes anuales ${ }^{4}$ — alrededor de 340 mil pesos a mitad del 2019-, es una necesidad, y no un lujo, contar con la participación de al menos dos de sus miembros en el mercado laboral (Osawa, 2000).

En estos casos, debido a que los incentivos están pensados para familias con ingresos medios y altos, se afecta negativamente a la contratación de mujeres casadas quienes no tienen la opción de decidir entre reingresar o no a la fuerza laboral. Y es en estos casos que su reingreso se limita a puestos de medio tiempo, con salarios mínimos y escasas o inexistentes prestaciones laborales. Asimismo, con el diseño institucional que se detalló líneas antes, dichas mujeres no tienen derecho a solicitar las deducciones familiares ni la exención en el pago de contribuciones para su fondo de pensiones. Es decir, las políticas para fomentar la "familia tradicional" no sólo afectan a las mujeres, sino particularmente a las mujeres que forman parte de familias con bajos ingresos.

En resumen, las políticas fiscales, de pensiones y laborales del Estado japonés han favorecido el modelo residual familista bajo el argumento de proteger a la familia japonesa. La realidad es que su fin es reducir gasto público en el cuidado de niños y ancianos, y contar con amortiguadores en el mercado laboral, garantizando la estabilidad en el empleo de hombres

\footnotetext{
${ }^{4}$ Como referencia, el índice de costo de vida "Big Mac" calculado por The Economist en Japón es de USD 3.5 (comparado con 2.57 en México). Otro referente es que, a mediados de 2019, en Japón la canasta básica mensual promediaba $¥ 40,000$, el transporte público rondaba los $¥ 10,000$ mensuales, y la renta por un departamento con servicios básicos de 30 metros cuadrados promediaba desde los $¥ 90,000$ en las zonas rurales y hasta los $¥ 140,000$ en las principales ciudades como Kioto, Osaka o Tokio.
} 
asalariados por medio de que las mujeres trabajen medio tiempo. En la siguiente sección se analizará la coyuntura demográfica que agrava estos elementos en perjuicio de las mujeres.

\section{Coyuntura demográfica}

A finales del siglo XX en Japón ${ }^{5}$, algunas mujeres menores de cuarenta años comenzaron a "centrarse en cumplir sus aspiraciones personales y profesionales y negarse a suscribirse a los patrones de fertilidad tradicionales" (Peng, 2012, p. 411). Los resultados de dichos cambios se reflejaron directamente en la tasa de fertilidad total (TFT) que desde 1989 descendió a 1.57 niños por mujer, muy por debajo de la necesaria tasa de reemplazo de 2.08 niños, y que ha continuado decreciendo hasta 1.41 niños por mujer para el año 2017 (Banco Mundial, 2018). Para el Estado japonés, esta coyuntura demográfica fue crítica por tres razones:

La primera razón tiene que ver con la Tasa de Dependencia (TD), que mide la relación entre los individuos económicamente activos —en este caso, el Estado japonés considera principalmente a los hombres asalariados_- y los económicamente inactivos — considerado el grupo de bebés, niños, ancianos y, según sea el caso, a las mujeres. Para que el modelo de bienestar familista ${ }^{6}$ funcione correctamente, se requiere una TD con un mayor número de personas económicamente activas quienes puedan mantener el bienestar e ingreso de aquellas otras económicamente inactivas. Esto no puede lograrse con una TFT tan baja y una correlativa esperanza de vida tan alta como la que Japón tiene actualmente ${ }^{7}$ (Ehara, 2013).

La segunda razón radica en la instrumentalidad de las mujeres para el modelo de bienestar familista. La función de las mujeres como "amas de casa de tiempo completo" —un eufemismo para la mujer cuidadora de niños y ancianos-, fue necesaria para evitar o al menos reducir el gasto social del gobierno durante la reconstrucción de la posguerra (Osawa, 2009). Tanto la política como las campañas en los medios de comunicación han alentado el

\footnotetext{
${ }^{5}$ Para un recuento más minucioso sobre la evolución de la población en la posguerra se sugiere el texto Japan's transition from the demographic bonus to the demographic onus (Ogawa, Kondo y Matsukura, 2005).

${ }^{6} \mathrm{Y}$ en general cualquier economía de mercado.

${ }^{7}$ La esperanza de vida en el último censo para Japón (2015) fue de 86.8 para mujeres y 80.5 para hombres, la tasa media combinada de 86 años es la más alta en el mundo (World Health Organization, 2016).
} 
ideal de la mujer como madre/esposa. De hecho, el modelo de "sostén familiar basado en el hombre" dependía desesperadamente de su correlativa "ama de casa de tiempo completo" no solo por la viabilidad de la familia, sino también por la del sistema de bienestar social en general. (Yoda, 2006, p.247)

Una tercera razón, se relaciona con el estallido de la burbuja en la economía japonesa a partir de los 90. Para la macroeconomía, tal estallido significó deflación crónica, bajo crecimiento y disminución de la producción bruta per cápita. Para efectos laborales, significó un incremento en el desempleo y más trabajadores a tiempo parcial quienes ganan a veces incluso menos que los salarios mínimos (Imai, 2011). Este último punto se relaciona directamente con la participación laboral de la mujer.

En términos absolutos, el gobierno de Japón presume una participación laboral de la mujer ${ }^{8}$ del 76.3\% (OECD, 2017a), representando el 43.32\% del total de la fuerza laboral en activo. Sin embargo, cuando se profundiza en estas cifras oficiales, se evidencia que la mujer en Japón tiene una escasa participación en puestos directivos (3.4\%) y parlamentarios $(9.5 \%)$; una brecha salarial de género respecto de empleos regulares del 25.7\% (OECD, 2017a) y de manera especialmente importante; ocupa $2 / 3$ del total de los empleos no regulares ${ }^{9}$ (OECD, 2017b). Esto quiere decir que la desigualdad laboral en perjuicio de la mujer no se ve tan claramente en el primer nivel de participación —cuantitativo-, sino en el de salarios, condiciones laborales y calidad de los empleos — cualitativo.

Con todos los elementos anteriores es posible mostrar la situación actual de la mujer japonesa en el mercado laboral. Por un lado, la coyuntura demográfica ha llevado a que varios políticos conservadores y ciertos sectores de la sociedad recriminen a las mujeres solteras ${ }^{10} \mathrm{y}$ divorciadas su "rompimiento con la institución de la familia por negarse a tener hijos o descuidar su crianza” (McCurry, 2018). Por otro lado, tras la continuada recesión económica que comenzó en 1990, su participación en el mercado laboral se vuelve necesaria tanto en

\footnotetext{
${ }^{8}$ Definida como la fracción de la población que trabaja o busca trabajo.

${ }^{9}$ Categoría que incluye al trabajador temporal, a tiempo parcial y subcontratado.

${ }^{10}$ Es importante señalar que, a diferencia de otros países industrializados, Japón tiene pocos hijos nacidos fuera del matrimonio (menos del 2.5\%) (OCDE, 2016).
} 
términos de productividad como de ingreso mínimo para la familia, si bien en condiciones marcadamente desiguales. Estas dos cuestiones representan el principal reto que tiene el gobierno japonés para el corto y mediano plazo. En la siguiente sección se describirá el marco jurídico con el que se ha tratado de enfrentar tal reto.

\section{Marco jurídico}

A finales del siglo XX, el gobierno japonés ideó una serie de reformas estructurales dirigidas a combatir los problemas laborales y demográficos descritos en las secciones anteriores. Más específicamente, se buscó: a) diseñar nuevas políticas de empleo y establecer estándares comunes en mujeres y hombres y; b) desarrollar prestaciones laborales con criterio de equidad y apoyos financieros para aumentar las tasas de natalidad y enfrentar la crisis demográfica (National Institute of Population and Social Security Research, 2014). Conforme con estos objetivos se promulgaron las siguientes normas:

a) Normas sobre igualdad de género en el ámbito laboral

En 1981 Japón firmó la Convención Internacional sobre la Eliminación de todas las Formas de Discriminación contra la Mujer (CEDAW). Sin embargo, el gobierno japonés no ha ratificado el protocolo opcional que permite, a cualquier individuo del país firmante, denunciar violaciones de la Convención. Con el argumento de que dicho protocolo "está en conflicto con el sistema judicial y la política legislativa de Japón”, se ha dejado a la CEDAW sin justiciabilidad, aunque con cierta influencia en la sociedad civil (Yamakawa,1999, p. $545)$.

A partir de tal influencia, diversos grupos de mujeres japonesas comenzaron a movilizarse y protestar contra las prácticas discriminatorias en materia de empleo y bienestar. Estos reclamos tuvieron como efecto paliativo la promulgación de la Ley para la Igualdad de Oportunidades en el Empleo (LIOE) en abril de 1986. Sin embargo, y a pesar de que la LIOE "prohibió la discriminación contra las mujeres en términos de capacitación, beneficios 
complementarios, jubilación obligatoria y despido", no lo hizo "en el reclutamiento, contratación, asignación y promoción” (Aspalter, 2016, p. 22). Esta omisión hizo que la LIOE resultara inútil en la práctica.

Asimismo, aunque la LIOE tuvo reformas en 1997 y, más recientemente en 2007, tampoco ha proporcionado incentivos fiscales para los empleadores que cumplen con la ley, ni ha prescrito multas o sanciones penales para las empresas con denuncias por discriminación sexual (Starich, 2010). Aún más importante, ni siquiera en su reforma de 2007, la ley se refiere o sanciona la discriminación indirecta, por lo cual queda impune la discriminación de salarios y ascensos basada en el sistema de dos vías — sogoshoku e ippanshoku- De esta forma, los derechos de igualdad y no discriminación tutelados por la LIOE no son justiciables y, de hecho, según Karen Starich: "Los tribunales japoneses consideran que la LIOE es simplemente una declaración política que ilustra las tendencias sociales actuales" (Starich, 2010, p.562).

Paralelamente, en 1999 se creó la Ley Básica para una Sociedad con Igualdad de Género (LBSIG), con la intención de aumentar la participación de la mujer en puestos de liderazgo. Asimismo, en 2005 se reformó dicha Ley para incluir indicadores y establecer el objetivo de alcanzar un 30\% de participación en tales puestos para 2020 (Geraghty, 2008, p. 523). Sin embargo, como en el caso de la LIOE, la falta de medidas coactivas, así como su lenguaje programático, la han hecho ineficiente para reducir la brecha laboral de género. Debido a ello, tanto la LIOE como la LBSIG han sido únicamente "fuentes simbólicas para mejorar la igualdad laboral que se han usado por el gobierno en turno" (Shire e Imai, 2000, p. 3).

Por último, hay que destacar que la Ley de Normas Laborales (LNL) es la regulación de referencia y aplicación general en materia laboral para Japón desde 1947. Con relación a la igualdad de género, la Ley ordena un trato igualitario para hombres y mujeres únicamente con respecto a los salarios (Artículo 4, LNL 1947, reformada en 2012). Sin embargo, no prohíbe la discriminación con respecto a la contratación, despido o promoción. En la práctica esto hace muy difícil que hombres y mujeres realicen exactamente el mismo trabajo y, por tanto, reciban exactamente el mismo salario (Starich, 2010). 
En síntesis, las tres principales leyes que se refieren a la igualdad de estándares en el empleo para hombres y mujeres han sido omisas, inaplicables o ineficientes.

b) Normas de equidad relacionadas con la crisis demográfica

Si bien la CEDAW también fue un referente para mejorar las condiciones laborales de la mujer, en realidad fue la crisis demográfica la que originó cambios sustantivos en el marco jurídico.

En este sentido, la LNL fue reformada en los años 80 para incluir limitaciones para despedir mujeres embarazadas (artículo 19), obligarlas a desempeñar trabajos peligrosos (artículo 64), y trabajar 6 semanas antes y después del parto (artículos 64 y 65 respectivamente). No obstante, estas disposiciones solamente se refieren al despido y no incluyeron una obligación positiva por parte del empleador. Bajo esta lógica, las mujeres no recibían sueldo ni prestaciones en el periodo en que se ausentaban y mucho menos tenían derecho a un subsidio o prestación por su estado de gravidez.

Esta cuestión implica una falta de equidad y, en última instancia, la imposibilidad de igualdad laboral para hombres y mujeres. Pero lo que resultaba más importante para el Estado japonés es que también se correlacionaba con la reducción en las tasas de natalidad. Debido a ello, en los años 90 se promulgaron leyes sobre licencias por maternidad y cuidado infantil.

La primera de estas leyes fue la Ley de Licencias por Maternidad (LLM), promulgada el 8 de mayo de 1991. La LLM originalmente permitía hasta un año de licencia, aunque sin sueldo, para las mujeres trabajadoras después del parto. Si bien no existía la obligación de pagar un sueldo en el periodo de licencia, sí obligaba al empleador a respetar el puesto de trabajo y la antigüedad en el momento de la reincorporación de la trabajadora con licencia.

A partir de una serie de reformas sustantivas a la LLM, se promulgó la Ley de Licencias de Maternidad y Cuidado de Infantes (LLMCI) en 2004. En esta nueva ley, el periodo de licencia se extendió hasta un año y medio y de forma innovadora, se hizo disponible para ambos sexos. Otra medida sin precedentes fue que por primera vez se obligó al empleador a pagar 
el 40\% del salario integrado durante los 6 primeros meses del periodo de licencia. Asimismo, se incluyó una disposición que permite a los empleados con hijos menores de la edad preescolar tomar hasta cinco días de licencia al mes, aunque en este caso, sin derecho a sueldo. Por último, a estos empleados se les dio el derecho de solicitar un límite en las horas extras, así como una exención del trabajo nocturno (LLMCI, 2004).

Aunque la LLMCI parecía progresista y una medida para la equidad de género, ésta ha hecho poco por cambiar los patrones sociales y empresariales. Por un lado, conforme con la información más reciente del Ministerio de Salud, Trabajo y Bienestar, sólo un 3\% de los hombres solicitan una licencia para el cuidado de infantes. Las razones de ello van desde los roles tradicionales de género, hasta las sanciones encubiertas del empleador que afectan promociones y ascensos en caso de solicitarla (Miyajima y Yamaguchi, 2017). Por otro lado, las empresas han interpretado que estas disposiciones solo aplican a los trabajadores ippanshoku (Nemoto, 2016). En consecuencia, muchas mujeres no acceden (y en algunos casos ni siquiera aspiran), a puestos directivos porque, en caso de querer tener hijos, no se beneficiarían de las disposiciones de la LLMCI.

Pero además de las fallas ontológicas de este marco jurídico, en su interpretación judicial han perdido aún más fuerza y eficacia. Así, por ejemplo, y a pesar del principio de igualdad del artículo 14 constitucional, los tribunales repetidamente han argumentado que no resulta aplicable al sector privado. En este sentido, aunque los jueces reconocen que la discriminación sexual en el salario o la promoción viola el artículo 14, suelen desestimar tales demandas en tanto que el empleador tiene "libertad de selección" conforme con la LNL (Nemoto, 2016). Esta interpretación se puede ver aplicada en los casos de discriminación sexual laboral contra Sumitomo Chemical Industries (2001), Showa Shell Co. (2009) y Chugoku Electric (2013), por citar los más recientes.

En definitiva, las leyes sin estímulos ni sanciones, las costumbres sociales y corporativas discriminatorias, así como la falta de una interpretación judicial con perspectiva de género, conllevan a que la segregación vertical por sexo siga estando arraigada en el marco jurídico japonés. 


\section{Conclusiones}

En diciembre de 2018, el Parlamento japonés aprobó una controversial ley para facilitar la entrada de inmigrantes trabajadores (The Asahi Shimbun, 2018). En su promulgación, Shinzo Abe indicó que el alto nivel de longevidad y la baja tasa de fertilidad, habían puesto a Japón en una debacle que hizo inevitable la apertura a la inmigración laboral. Si bien la ley busca atraer principalmente mano de obra "semi calificada" para sectores como el de la construcción, hotelero, limpieza y cuidado de ancianos, la pregunta que surge de inmediato es ¿qué hay de las trabajadoras japonesas?

En este artículo explicamos cómo es que dos construcciones del Estado japonés, la familia tradicional y la buena madre/esposa sabia, fueron utilizadas para facilitar la modernización a finales del siglo XIX y su reconstrucción en la posguerra. El diseño de Japón como un estado residual familista hizo que, en estos dos momentos históricos, tales construcciones fueran eficientes, aunque discriminatorias hacia la mujer. No obstante, cuando se tuvo que enfrentar la crisis económica de los 90, y a partir de la liberalización del mercado laboral, ambas construcciones dejaron de ser viables.

Algo similar ocurrió respecto al diseño institucional que buscaba perpetuar el rol de mujeres como amas de casa de tiempo completo. Por varias décadas se privilegió una visión monolítica de la familia por medio de exenciones fiscales y estímulos en el sistema de seguridad social y pensiones. Esto, sin embargo, se volvió insostenible cuando las condiciones económicas hicieron patente que, contrario a la creencia popular, no todas las familias japonesas son de clase media ni sustentadas por el padre de familia.

Por si los anteriores dos componentes no fueran suficientes, la coyuntura demográfica fue una manifestación tangible de que las construcciones del Estado japonés habían perdido toda validez. La baja tasa de fertilidad, limitada productividad, escasa mano de obra y segregación laboral son, todos ellos, problemas vinculados con arquetipos y estructuras caducas. En la realidad del Japón actual, ya no cabe la pregunta de si es o no deseable incluir a la mujer en el mercado laboral sino cómo hacerlo más rápido, eficiente y equitativamente posible. 
En este sentido, el marco jurídico y la interpretación judicial podrían ser un motor para producir las transformaciones que los tres componentes anteriores no han podido articular. Empero, hemos visto cómo normas ineficaces y jueces conservadores en realidad han cimentado el estatus, salario y prestaciones precarias para las mujeres. Aún más importante, al igual que la forma en la cual se han abordado los tres componentes anteriores, el derecho japonés no ha podido —o querido— reconocer la realidad social, empresarial y laboral más allá de las construcciones obsoletas.

Para responder a la pregunta con la que se inició esta sección, ¿qué hay de las trabajadoras japonesas?, es necesario tomar una perspectiva que considere en su conjunto e interacción los cuatro temas analizados en este artículo - antecedentes, diseño institucional, coyuntura demográfica y marco jurídico- Si bien la apertura hacia la inmigración laboral resulta necesaria e ineludible, también lo es la equidad laboral para las mujeres japonesas. No basta con resultados que cuantitativamente suenen incluyentes, sino con acciones y resultados que cualitativamente lo sean.

En este sentido, el primer paso es reconocer que la mujer japonesa puede ser buena madre y esposa sabia, pero no tiene que serlo. Los programas que únicamente favorecen a mujeres como amas de casa de tiempo completo, deben dar paso a otros que busquen la participación equitativa en el trabajo, fomenten el equilibrio entre el trabajo y la vida personal y den alternativas de contratación, capacitación y ascenso sin segregación por género. No se trata de eliminar a un determinado modelo de familia, sino de reconocer que hay múltiples de ellos y muy diversas formas de participación de la mujer.

Un segundo paso implica reformar el diseño institucional para ser equitativo y facilitar diversas formas de trabajo para las mujeres; siempre con miras hacia una igualdad sustancial. Las cuotas de género y acciones afirmativas, sistemas de guarderías y cuidado de ancianos eficientes, así como programas fiscales y de seguridad social incluyentes y equilibrados son medidas concretas que pueden enraizarse en el estado de bienestar japonés si se apoyan debidamente. 
La forma de abordar la coyuntura demográfica sería un tercer paso para favorecer la equidad de género en Japón. La crisis en tasas de fertilidad y productividad son una responsabilidad compartida por gobierno y sociedad civil, así como por mujeres y hombres. Aún más, resulta insuficiente una visión de esta crisis como un problema por resolver, y más bien se requiere ponderar las diversas causas, próximas y mediatas, para plantear debidamente el problema antes de buscar soluciones. En este sentido, las políticas públicas laborales pueden ser uno de los elementos que ayudarían a la situación demográfica, pero no son necesariamente los únicos o siquiera los más importantes.

Por último, el derecho japonés debe ser un instrumento no sólo para la modernización y estabilidad de la economía, sino también para la justicia e igualdad entre las personas. Tomando la realidad social como base, tanto leyes como jueces deben servir para cuestionar y no solamente legitimar el statu quo. Es necesario que el derecho sirva de contraste y contrapeso a los discursos, instituciones y políticas públicas, actualizando y, en su caso, refutando, los planteamientos de políticos y burócratas japoneses.

Aunque esta tarea requiere de estudios y propuestas más detalladas de las que se ofrecen en este artículo, la equidad laboral en Japón no sólo es un tema prioritario y conveniente para las mujeres, sino indispensable para la propia subsistencia del Estado japonés. Consecuentemente, este texto buscó dar una visión panorámica que reconozca la complejidad del tema y propicie otros estudios sobre el mismo. 


\section{Referencias bibliográficas}

Akabayashi, Hideo. (2006). The labor supply of married women and spousal tax deductions in Japan. Review of Economic Household, 4(4), 349-378. doi: 10.1007/s11150-006-00125

Aspalter, Christian. (2016). Christian Social Works in East Asia. New York: Routledge.

Banco Mundial. (2018). Tasa de fertilidad, total (nacimientos por cada mujer), Recuperado de https://datos.bancomundial.org/indicador/sp.dyn.tfrt.in

Bessho, Shun-ichiro y Hayashi, Masayoshi. (2014). Intensive margins, extensive margins, and spousal allowances in the Japanese system of personal income taxes: a discrete choice analysis. Japan International. Economics, 34, 162-178. doi: https://doi.org/10.1016/j.jjie.2014.06.001

Bishop, Beverley. (2012). Globalisation and Women in the Japanese Workforce. Londres: Routledge.

Constitución de Japón, (3 de mayo de 1947).

Corte de Distrito de Osaka. (27 de junio de 2001). Caso Sumitomo Chemical Industries. Rodo Hanrei [Precedente de Derecho Laboral] 809-5, publicado en Roudo houristy jyunpou [Gaceta Judicial].

Corte de Distrito de Tokio. (29 de junio de 2009). Showa Shell Co. Rodo Hanrei [Precedente de Derecho Laboral] 846-10, publicado en Roudo houristy jyunpou [Gaceta Laboral Judicial].

Ehara, Yumiko. (2013). Japanese Feminist Social Theory. En Anthony Elliott, Katagiri Masataka y Atsushi Sawai (Eds.), The Routledge Companion to Contemporary Japanese 
Social Theory: From individualization to globalization in Japan today (pp.162-175). New York: Routledge.

Fujimura-Fanselow, Kumiko. (1991). The Japanese Ideology of Good Wives and Wise Mothers: Trends in Contemporary Research. Gender and History, 3(3), 345-349.

Gender Equality Bureau. (2017). Gender Equality Policy in Japan. Cabinet Office, Government of Japan. Recuperado de: http://www.gender.go.jp/international/int_kaigi/int_acw3/pdf/contry_presentation_japan -09.pdf

Geraghty, Kristina. (2008). Taming the Paper Tiger: A Comparative Approach to Reforming Japanese Gender Equality Laws. Cornell International Law Journal, 41(2), 503-542.

Hobsbawm, Eric y Ranger, Terence. (2002). La invención de la Tradición. Barcelona: Crítica.

Hozumi, Nobushige. (1912). Lectures on the New Japanese Civil Code as Material for the Study of Comparative Jurisprudence. Tokio: Maruzen Kanushiki-Kaisha.

Imai, Jun. (2011). The Transformation of Japanese Employment Relations: Reform without Labor. London: Palgrave MacMillan.

Johnson, Chalmers. (1982). MITI and the Japanese Miracle, Stanford: Stanford University Press.

Kamimura, Yasushiro y Soma, Naoko. (2013). Active labor market policies in Japan: a shift away from the company-centered model? Journal of Asian Public Policy, 6(1), 42-59. 
Kasza, Gregory. (2011). The Rise (and fall?) of Social Equality: The Evolution of Japan's Welfare State. En Alisa Gaunder (Ed.), Routledge Handbook of Japanese Politics. (pp. 189-200). New York: Routledge.

Nemoto, Kumiko. (2016). Too Few Women at the Top: The Persistence of Inequality in Japan. Ithaca: Cornell University Press.

Labor ministry admits to cover-up in wage statistic scandal. The Asahi Shimbun. (2 de febrero de 2019) Recuperado de http://www.asahi.com/ajw/articles/AJ201902020039.html

Law on foreign labor still a policy riddled with huge holes. (8 de diciembre de 2018). The Asahi Shimbun.

Ley de Licencias de Maternidad y Cuidado de Infantes (3 de mayo de 2004).

Lewis, Jane. (1992). Gender and the Development of Welfare Regimes. Journal of European Social Policy, 1(2), 93-105.

Maki, John. (1968). Japanese Constitutional Style. En Dan Henderson (Ed.), The Constitution of Japan: Its First Twenty Years, 1946- 67. (pp.323-358) Seattle: University of Washington Press.

Mari, Miura. (2012). Welfare Through Work: Conservative Ideas, Partisan Dynamics, and Social Protection in Japan. Ithaca: Cornell University Press.

McCurry, Joseph. (11 de mayo de 2018). Mujeres solteras una carga para el Estado, dice el Parlamentario Japonés Kanji Kato [Single women a "burden on the state", says Japanese MP Kanji Kato]. The Guardian.

Ministerio del Interior. (2015). Encuesta sobre fuerza laboral 2015. Recuperado de: http://www.stat.go.jp/data/roudou/sokuhou/4hanki/dt/index.htm 
Ministry of Health Labor and Welfare. (2018). White Paper on Labor 2018. Tokyo: Ministry of Labor.

Miyajima, Takeru y Yamaguchi, Hiroyuki. (2017). I Want to but I Won't: Pluralistic Ignorance Inhibits Intentions to Take Paternity Leave in Japan. Frontiers in Psychology. 8, 1-12 Recuperado de: https://www.frontiersin.org/articles/10.3389/fpsyg.2017.01508/full

Mizubayashi, Takashi. (1987). “明治国家のいえ” (Meiji kokka no ie/ La “familia” en el Estado Meiji), Historia de Japón, 2, 382-390.

Murakami, Yasusuke. (1984). Ie society as a pattern of civilization. The Journal of Japanese Studies, 10(1), 279-363.

Nakamura, Kichisaburo. (1962). The Formation of Modern Japan as viewed from Legal History. Tokyo: The Centre for East Asian Cultural Studies.

Soma, Naoko y Yamashita, Jun. (2011). Child Care and Elderly Care Regimes in Japan. Journal of Comparative Social Welfare, 27(2), 133-156.

Shire, Karen y Imai, Jun. (2000). Flexible equality: men and women in employment in Japan. Working Papers on East Asian Studies 30/2000, University of Duisburg-Essen, Institute of East Asian Studies IN-EAST.

National Institute of Population and Social Security Research. (2014). Social Security in Japan. Recuperado de: http://www.ipss.go.jp/.

Ogawa, Kondo y Matsukura, Rikiya (2005). Japan's Transition from the Demographic Bonus to the Demographic Onus. Asian Population Studies, 1(2), 207-226. 
Organisation for Economic Co-operation and Development (OECD). (2012). Closing the Gender Gap: Act Now. Paris: OECD Publishing.

Organisation for Economic Co-operation and Development (OECD). (2016). Family Database. Maternal Employment by partnership status. Recuperado de: http://www.oecd.org/els/soc/LMF_1_3_Maternal_employment_by_partnership_status.p df

Organisation for Economic Co-operation and Development (OECD). (2017a). The Pursuit of Gender Equality: An Uphill Battle. Paris: OECD Publishing.

Organisation for Economic Co-operation and Development (OECD). (2017b). Japan Policy Brief. Policy Briefs. Abril, 2017 Recuperado de: https:/www.oecd.org/japan/japanstrenghtening-innovation-for-productivity-and-greater-wellbeing.pdf

Osawa, Mari. (2000). Government Approaches to Gender Equality in the mid-1990s. Social Science Japan Journal 3(1), 3-19.

Osawa, Mari. (2009). Twelve Million Full-time Housewives: The Gender Consequences of Japan's Post-War Social Contract. En Olivier Zunz, Leonard Schoppa y Nobuhiro Hiwatari. (Eds.), Social Contracts under Stress: The Middle Classes of America, Europe and Japan at the Turn of the Century. (pp. 265-287). New York: Russel Sage Foundation.

Osawa, Mari. (2013). Governance of Livelihood Security. Tokyo: Yukihaku.

Peng, Ito. (2012). Social Care in Crisis: Gender, Demography, and Welfare State Restructuring in Japan Social Politics. Social Politics, 9(3), 411-443.

Ranstad Workmonitor. (2019). $1^{\text {st }}$ Quarter Data: Recuperado de: https://www.randstad.com/workforce-insights/global-hr-research/randstad-workmonitor/

Röhl, Wilhelm. (2005). History of Law in Japan since 1868. Leiden: Brill. 
Sirota, Gordon; Pharr, Susan; Molony, Barbara y Hastings, Sally. (1988). Celebrating Women's Rights in the Japanese Constitution. U.S. - Japan Women's Journal, 14, 64-83. Recuperado de https://www.jstor.org/stable/42772126

Starich, Megan. (2010). Comment: The 2006 Revisions to Japan's Equal Opportunity Employment Law - A Narrow Approach to a Pervasive Problem. Annual review of Sociology, 36, 225-247.

Steenstrup, Carl. (1991). German Reception of Roman Law and Japanese Reception of German Law. Intercultural Communication Studies, 1(1), 273-293.

Suprema Corte de Japón. (10 de marzo de 2013). Caso Chugoku Electric Power Co. Caso 2327, publicado en Roudo houristy jyunpou [Gaceta Laboral Judicial].

Tachibanaki, Toshiaki. (2010). The New Paradox for Japanese Women: Greater Choice, Greater Inequality. Tokyo: International House of Japan.

Takahashi, Shingo. (Mayo 28 de 2010). A structural estimation of the effects of spousal tax deduction and social security system on the labor supply of Japanese married women. Tokyo Labor Economics Seminar.

Titmuss, Richard. (2008). Social Policy. En Stephan Leibfriedy y Steffen Mau. (Eds.), Welfare States: Construction, Deconstruction, Reconstruction. Cheltenham: Edward Elgar Publishing.

Ueno, Chizuko. (2008). The Modern Family in Japan: its Rise and Fall. Melbourne: Trans Pacific Press.

World Health Organization. (2016). World Health Statistics 2016: Monitoring health for the SDGs Annex B: tables of health statistics by country, WHO region and globally. 
Recuperado de:

https://www.who.int/gho/publications/world_health_statistics/2016/Annex_B/

Wilthagen, Ton y Tros, Frank (2004). The Concept of "Flexicurity": a new approach to regulating employment and labour markets. European Review of Labour and Research, 10 (2), 166-186. doi: https://doi.org/10.1177/102425890401000204

Yajima, Daisuke (14 de diciembre de 2018). Ministry finds that 10 medical schools rigged entrance exams. The Asahi Shimbun.

Yamaguchi, Kambayashi y Higuchi, Yamamoto. (2008). Ronso: Work-life Balance in Japan. Tokyo: Nihon Keizai Shimbunsha.

Yamakawa, Ryuichi. (1999). We've Only Just Begun: The Law of Sexual Harassment in Japan. Hastings International and Comparative Law Review, 22(3), 253-270.

Yoda, Tomiko. (2006). The Rise and Fall of Maternal Society: Gender, Labor and Capital in Contemporary Japan. En Tomiko Yoda y Harry Harootunian. (Eds.), Japan after Japan: Social and Cultural Life from the Recessionary 1990s to the Present. (pp. 239-274). Durham: Duke University Press.

Yokoyama, Izumi. (2018). How the tax reform on the special exemption for spouse affected the workhour distribution. Journal of the Japanese and International Economies, 49(3), 69-84. doi: https://doi.org/10.1016/j.jjie.2018.04.002

\section{Sobre el Autor}

Abogado por la Escuela Libre de Derecho, maestro en estudios de Asia y África con especialidad en Japón por El Colegio de México y doctor en derecho por University of British Columbia, Vancouver. Profesor en el Centro de Estudios de Asia y África de El Colegio de México e Investigador en la Escuela Libre de Derecho. Becario en maestría y doctorado por 
Japan Foundation e investigador invitado por la Universidad Waseda, Tokio (2012-2013). Líneas de investigación: derecho japonés; sociedad y derecho; constitucionalismo comparado; tecnología y derecho. 